\title{
Impacto económico de los asistentes a la Universiada de Invierno del 2015 en la ciudad de Granada
}

\author{
Economic impact of the attendees to the Winter \\ Universiade 2015 in the city of Granada
}

\author{
Antonio Roca-Cruz ${ }^{1 *}$, Josué González-Ruiz ${ }^{1}$ Pedro Porcel-Rodríguez ${ }^{2}$ y David Cabello-Manrique \\ ${ }^{1}$ Departamento de Educación Física y Deportiva, Universidad de Granada (España) \\ ${ }^{2}$ Departamento de la Didáctica de la Expresión Musical, Plástica y Corporal, Universidad de Jaén (España)
}

Resumen: Los mega-eventos deportivos crean una nueva imagen del país anfitrión, atrae a espectadores y a los medios de comunicación (Santo, 2005; Cornelissen y Swart, 2006). El impacto económico de un evento puede definirse como el cambio económico neto en el país anfitrión que deriva del gasto atribuido al evento (Crompton, 1995). Ya que uno de los beneficios más importantes son los beneficios permanentes en la ciudad (Witt, 1988) como la mejora de las instalaciones o la imagen del país. En relación al turismo de un gran evento hay un moderado incremento del turismo interno de negocios en una ciudad durante el desarrollo del evento (OECD, 2012). Por lo tanto, la ciudad aumenta su potencial de inversión y actividad comercial (Avison Young, 2003) El objetivo del presente estudio fue analizar el impacto económico generado por los asistentes en la ciudad de Granada durante la Universiada Granada 2015.

Palabras clave: Evento deportivo, Impacto económico, Satisfaccion
Abstract: Mega-sport events create a new image of the host country, attracting viewers and the media (Santo, 2005; Cornelissen and Swart, 2006). The economic impact of an event can be defined as the net economic change in the host country that derives from the expenditure attributed to the event (Crompton, 1995). Since one of the most important benefits are the permanent benefits in the city (Witt, 1988) as the improvement of the facilities or the image of the country. In relation to the tourism of a big event there is a moderate increase of the internal business tourism in a city during the development of the event (OECD, 2012). Thus, the city increases its investment potential and commercial activity (Avison Young, 2003). The objective of the present study was to analyze the economic impact generated by the attendees in the city of Granada during the Universiade Granada 2015

Keywords: Sport event, Economic impact, Satisfaction.

\section{Introducción}

Los mega-eventos deportivos crean una nueva imagen del país anfitrión, atrae a espectadores y a los medios de comunicación (Santo, 2005; Cornelissen y Swart, 2006). El impacto económico de un evento puede definirse como el cambio económico neto en el país anfitrión que deriva del gasto atribuido al evento (Crompton, 1995). Ya que uno de los beneficios más importantes son los beneficios permanentes en la ciudad (Witt, 1988) como la mejora de las instalaciones o la imagen del país. En relación al turismo de un gran evento hay un moderado incremento del turismo interno de negocios en una ciudad durante el desarrollo del evento (OECD, 2012). Por lo tanto, la ciudad aumenta su potencial de inversión y actividad comercial (Avison Young, 2003).

Estos eventos deportivos, al igual que otras actividades, pueden ser categorizados como servicios, al presentar consumo intangible, heterogéneo, producido de forma simultánea, siendo consumidos y perecederos (Bamford y Dehe, 2016). Además, se está produciendo una diferenciación del público en la forma en la que asisten y acuden a ver los eventos

Dirección para correspondencia [Correspondence address]: Antonio Roca-Cruz. Departamento de Educación Física y Deportiva, Universidad de Granada (España). E-mail: antonioroca@ugr.es deportivos parece estar afectando progresivamente tanto la forma en que las personas apoyan y siguen al deporte y su sentido de pertenencia social y de identificación (Bouchet et al., 2011).

La Universiada es un evento deportivo de carácter internacional que cuenta con casi 100 años de historia (World Student Games - París 1923) y en el que se reúnen unos 10.000 deportistas, en las de verano, y 3.000 para las de invierno. El evento sigue, prácticamente, la misma estructura de celebración y organización de unos Juegos Olímpicos (JJOO), cuyo modelo utiliza como referencia. Compiten diversos países en unos determinados deportes, considerados olímpicos, con el objetivo de conseguir acceder al podio y colgarse una de las tres medallas que refrendan el esfuerzo invertido. Pero esta cita tiene un aspecto particular que la diferencia de unos JJOO, y es que sus participantes son universitarios, quienes representan a los respectivos países en función de la universidad en la que se encuadra cada uno de ellos.

Esta Universiada se compone de dos tipos de modalidades, una de invierno y otra de verano, las cuales se celebran, conjuntamente, cada dos ańos en diversos lugares del mundo. En el año 2015, Espańa, por cuarta vez en su historia (una de verano y 3 de invierno), acogió esta cita en la ciudad 
de Granada, junto con Strbske Pleso/Osrblie (Eslovaquia), la sede encargada de celebrar y organizar el acontecimiento deportivo.

Es evidente que la puesta en marcha de una cita como ésta supone un amplio e intenso despliegue de capital humano y financiero capaz de soportar la construcción y adaptación de instalaciones deportivas, mantenimiento de los deportistas participantes y sus equipos de apoyo o la constitución de un personal organizador, entre otras. Un gasto que cualquier ciudad, ya sea apoyada económicamente o no, debe de afrontar para que su celebración genere las mayores garantías de éxito. Los eventos (culturales, artísticos o deportivos) crean una gran oportunidad para el desarrollo turístico de ciudades, regiones o países incentivando el desarrollo socioeconómico local, ayudando a la creación de empleo, y a la creación y mejora de las infraestructuras (carreteras, hoteles, restaurantes, comercios, etc.). Todo esto beneficia los turistas y la población local (Fernández, 2014).

Pero resulta interesante observar que son muchas las ciudades candidatas a acoger este tipo de citas. Si su celebración supone un coste tan elevado ¿por qué hay demanda e interés por parte de los diferentes entes públicos en ofrecerse como sede para la recepción de una Universiada?. Esto tiene su explicación en los efectos multiplicadores generados en la economía con motivo de la inversión realizada en un sector concreto, como en el caso de Barcelona 92 donde el impacto de la nominación olímpica fue inmediato: el paro inició un acusado descenso, el mercado de la vivien- da se reanimó y la construcción alcanzó su punto máximo (Brunet, 1995).

El creciente interés de los organismos públicos en celebrar estos eventos reside en la creencia general, en gran parte de tipo económica, de la positiva repercusión que tiene en el resto de sectores económicos una inversión satisfecha en uno de ellos. Esto procede de la relación inter-industrial existente entre las diferentes ramas de actividad que intervienen en la economía y que provocan efectos multiplicadores entre ellas como bien muestran instrumentos como el marco InputOutput (Leontief, 1951).

El principal interés del presente proyecto consiste en demostrar y calcular los efectos que se han producido en la economía de Granada con motivo de la celebración de la Universidad Invierno Granada 2015. Así se podrá conocer la repercusión que ha tenido este gasto realizado en variables como la producción o el empleo en las distintas ramas de actividad que componen la economía.

Los eventos deportivos según Lopes y Ricci (2016) producen legados, estos legados positivos pueden ser: producción de infraestructura; beneficios a grupos de interés; generación de empleos; nuevos valores sumados al Producto Interno Bruto (PIB); el aumento de la demanda turística, mejora de la imagen del país y trae orgullo e involucra la población. Otro punto positivo de acuerdo con (Silva, 2007) es que un mega evento involucra toda la ciudad, puede servir de estímulo a los jóvenes y proporciona una mayor presencia en el deporte (Tabla 1),

Tabla 1. Impactos positivos de eventos a gran escala.

\begin{tabular}{ll}
\hline a) Impactos de evento & Construcciones deportivas, estadios, arenas y otros equipamientos. \\
b) Impactos de la candidatura de evento & Proceso, organización de evento, planificación y proyectos \\
c) Impactos de imagen del País & El país se vuelve mas conocido en el panorama internacional. \\
d) Impactos de gobierno & Planificación participativa, colaboración del sector publico y el sector privado \\
e) Impactos de conocimiento & Entrenamiento y capacitación profesional \\
\hline
\end{tabular}
Fuente: Adaptado de Tavares (2011)

El objetivo del presente estudio fue analizar el impacto económico generado por los asistentes durante la Universiada de Invierno - Granada 2015 en la ciudad de Granada.

\section{Método}

El estudio realizado se centra en el análisis de los efectos económicos derivados de la celebración, en el municipio de Granada de la Universiada de Invierno 2015 entre las fechas del 4 y 14 de febrero del 2015. El espacio territorial de referencia es municipio de Granada, ya que aunque algunas pruebas se realizaban en la estación de esquí situada a $30 \mathrm{~km}$ de Granada, todos los atletas y asistentes se alojaban en la Capital. Lo que realmente es relevante y de interés, es el análisis del gastos y patrocinios hechos por las personas no residentes en Granada

Para poder medir este tipo de efectos, la economía dispone de diversas herramientas con las que poder obtener un acercamiento certero de este cálculo. Existen múltiples propuestas metodológicas para llevar a cabo este tipo de análisis. Según Barajas, Salgado y Sánchez (2012), entre los métodos de análisis del impacto socioeconómico más frecuentemente empleados en el ámbito del deporte se encuentran las Cuentas Satélite, las Tablas Input-Output (TIO) o el Análisis Coste-Beneficio (ACB). Otros autores como Sanz e Insúa (2003) también incluyen métodos como la Tabla Económica de Conjunto (TEC), así como los análisis sectoriales y regionales, mientras que Fernández (2014) tiene en cuenta el modelo de equilibrio general computable (EGC). 
Dado que el ACB constituye un método más amplio y flexible para identificar todas las posibles ganancias y pérdidas de un proyecto, a la vez que evalúa las repercusiones socioeconómicas, y que el análisis Input-Output (AIO) cuantifica de forma precisa la repercusión económica en el resto de sectores de la economía de un proyecto realizado en una rama concreta, para conseguir la imagen más fiel posible, en el presente informe se han tenido en cuenta ambos métodos para medir el impacto económico de la Universiada, adaptando su técnica al contexto preciso en el que se desarrolla.

La aplicación del ACB, en este tipo de análisis, puede ser de utilidad para identificar los diferentes gastos e ingresos generados durante el evento con los que, posteriormente, se podrá construir el vector columna necesario para la aplicación del modelo de demanda. Este permitirá conocer el efecto en los demás sectores de la economía, tanto en producción como en empleo.

\subsection{Participantes}

Participaron en este estudio 670 sujetos, 440 varones y 230 mujeres, procedentes de 6 nacionalidades distintas como España, Canadá, Estados Unidos, Eslovaquia, Rusia y China.

\subsection{Procedimiento}

Se pasó un cuestionario en persona, a los asistentes en las instalaciones de las competiciones deportivas del evento en los últimos días para comprobar los gastos de alojamiento, restaurantes, transportes, alimentación, tours y otros durante el evento. Los datos fueron aprobados por la Concejalía de Deportes del Ayuntamiento de Granada.

La información necesaria para la realización del estudio sobre el impacto económico de la organización de la Universiada de Invierno 2015 en el mes de febrero de 2015 se consiguió mediante la realización de entrevistas a los organizadores y una encuesta a los asistentes, ya fuera como participantes o como espectadores.

Por otro lado, desde la organización se facilitaron los datos solicitados y respondieron a las cuestiones que se le realizaron.
Los espectadores fueron encuestados por un equipo de encuestadores de la propia organización del evento entrenados para la ocasión, en los días del evento, en las instalaciones donde se realizó la competición. En total, se encuestaron 670 personas lo que implica una margen de error del 5\% con un nivel de confianza del 95\%. Para garantizar la aleatoriedad de la encuesta, los encuestadores siguieron un patrón de conducta dirigiéndose a una persona de cada tres en cada zona y realizándolas en diversos momentos se estructura en cuatro partes diferenciadas con un total de 26 preguntas. La primera parte del cuestionario aborda la identificación en términos deportivos de la persona encuestada, entre las que destaca su condición de participante en la prueba que es ese momento actua como espectador o sólo espectador y, en caso de que participe, el país de pertenencia.

La segunda parte del cuestionario se basa en el modelo EVENTQUAL (Calabuig y Crespo, 2009). La escala utilizada en este trabajo, ha mostrado buenos índices de fiabilidad y validez durante la celebración de un megaevento deportivo de carácter multideportivo como es nuestro caso

\subsection{Análisis estadístico}

Los resultados del estudio aparecen como media y desviación típica y el valor de significatividad. Se analizó la t de student de los aspectos sociométricas diferenciados por género. Así, como los estadísticos descriptivos y el análisis de frecuencias de las variables del estudio. El nivel de confianza fue 95 por ciento y se empleó el paquete estadístico SPSS, software versión 19.0 para Windows (SPSS, Armonk, New York)

\section{Resultados}

Los resultados de las variables del estudio obtenidos mediante la prueba $t$ de Student para muestras independientes se pueden observar en la tabla 2, con una media de edad de los asistentes de 27 ańos con los gastos mas elevados en alojamiento con 492,83 y en restauración 226,66 que junto con otros gastos supusieron un gasto medio de 185,66 euros.

Tabla 2. Edad (años) y gastos (euros) segmentados por género. Los datos se presentan como media y desviación típica (DT) en porcentajes.

\begin{tabular}{lcccccc}
\hline & \multicolumn{2}{c}{$\begin{array}{c}\text { Todos } \\
(\mathbf{n}=\mathbf{6 7 0})\end{array}$} & \multicolumn{2}{c}{$\begin{array}{c}\text { Varones } \\
(\mathbf{n}=\mathbf{4 4 0})\end{array}$} & \multicolumn{2}{c}{$\begin{array}{c}\text { Mujeres } \\
(\mathbf{n}=\mathbf{2 3 0})\end{array}$} \\
\hline Variables & Media & DT (\%) & Media & DT (\%) & Media & DT (\%) \\
\hline Edad & 27 & 0,73 & 30 & 0,69 & 24 & 0,77 \\
Gasto alojamiento & 492,83 & 712,0 & 370,00 & 535,4 & 615,65 & 888,6 \\
Gasto restaurante & 226,66 & 366,1 & 236,36 & 375,1 & 216,96 & 357,1 \\
Gasto transporte & 188,87 & 301,0 & 120,34 & 220,6 & 257,39 & 381,4 \\
Gasto transporte privado & 42,50 & 54,0 & 34,77 & 44,7 & 50,22 & 63,2 \\
\hline
\end{tabular}




\begin{tabular}{lcccccc}
\hline & \multicolumn{2}{c}{$\begin{array}{c}\text { Todos } \\
(\mathbf{n}=\mathbf{6 7 0})\end{array}$} & \multicolumn{2}{c}{$\begin{array}{c}\text { Varones } \\
(\mathbf{n}=\mathbf{4 4 0 )}\end{array}$} & \multicolumn{2}{c}{$\begin{array}{c}\text { Mujeres } \\
(\mathbf{n}=\mathbf{2 3 0})\end{array}$} \\
\hline Variables & Media & DT $(\%)$ & Media & DT (\%) & Media & DT (\%) \\
\hline Gasto comida & 47,62 & 18,6 & 48,07 & 21,1 & 47,17 & 16,1 \\
\hline Gasto otros & 248,37 & 273,4 & 207,61 & 253,0 & 289,13 & 293,8 \\
\hline Gasto tours & 52,77 & 82,7 & 37,27 & 73,9 & 68,26 & 91,5 \\
\hline Gasto medio & 185,66 & 193,1 & 150,63 & 165,6 & 220,68 & 234,4 \\
\hline
\end{tabular}

En la tabla 3, se puede apreciar el gasto medio por países, destacando EE.UU, que supera 10 veces el gasto realizado por los asistentes españoles.

Tabla 3. Gasto medio (euros) de cada país.

\begin{tabular}{lcccccc}
\hline & $\begin{array}{c}\text { Canadá } \\
(\mathrm{N}=90)\end{array}$ & $\begin{array}{c}\text { EEUU } \\
(\mathrm{N}=110)\end{array}$ & $\begin{array}{c}\text { Eslovaquia } \\
(\mathrm{N}=20)\end{array}$ & $\begin{array}{c}\text { Rusia } \\
(\mathrm{N}=30)\end{array}$ & $\begin{array}{c}\text { China } \\
(\mathrm{N}=20)\end{array}$ & $\begin{array}{c}\text { España } \\
(\mathrm{N}=400)\end{array}$ \\
\hline Gasto medio & 370,32 & 407,08 & 301,43 & 383,10 & 298,93 & 38,57 \\
\hline
\end{tabular}

El gasto de alojamiento es el más elevado con un gasto medio de 492,83 euros (tabla 2) ya que el evento deportivo tuvo una duración de 14 días y los asistentes nacionalizados fuera de España gastaron mayor cantidad de dinero como por ejemplo EEUU que gastó una media de 407,08 euros o en el caso de Rusia 383,10 euros. España gastó de media 38,57 euros (tabla 3).

\section{Discusión}

La celebración de un gran evento deportivo en una ciudad supone una oportunidad única para la transformación y el relanzamiento de la misma. Las ciudades sede suelen sufrir una evolución tal que en unos pocos años se puede conseguir incrementar la calidad de vida de los ciudadanos más de lo que normalmente se hubiese conseguido en decenios (Mundina y Calabuig, 2011).

Por tal motivo estas grandes ciudades deben de buscar no solo el éxito a la hora de ser elegida como sede del gran evento, como es el caso de la Copa del América en Valencia (Jones, 2001), si no también, y de acuerdo con la orientación al ciudadano, deben satisfacer las necesidades que demandan sus ciudadanos y organizaciones. Estas necesidades pueden referirse a la mejora de infraestructuras y equipamientos, la disminución de la contaminación, el aumento de dotaciones de uso social, la creación de empleo estable y la mejora de la seguridad ciudadana. Y por supuesto un objetivo prioritario es la satisfacción de los espectadores del propio evento deportivo, con lo cual, el objetivo inicial - conseguir la sede del evento - debe de transformarse en la satisfacción de los ciudadanos que anteriormente han legitimado de facto la solicitud de sede del evento (Mundina y Calabuig, 2011).

Desde el punto de vista del análisis del deportista, desde esta perspectiva, ha sido poco estudiado (Theodorakis y Bebetsos, 2003) a pesar de ser el elemento fundamental en los eventos deportivos. Con ello se pueden obtener indicadores de la gestión y ser tenidos en cuenta para la solicitud de otros eventos deportivos, o incluso puede utilizarse para ser comparado con otras ciudades candidatas o sedes.

El evento deportivo ha creado una nueva imagen de la ciudad atrayendo a espectadores y medios de comunicación (Santo, 2005; Cornelissen y Swart, 2006). Podemos decir que la ciudad de Granada ha aumentado su potencial de inversión y actividad comercial afirmando lo que exponía el estudio de Avison Young (2003) . Aunque los beneficios más importantes son los beneficios permanentes en la ciudad (Witt, 1988). Estos resultados pueden servir de guía para conocer el gasto de todos los asistentes a la Universiada Granada 2015 y ayudar a los gestores a saber posicionar los eventos deportivos según los condicionantes turísticos del lugar de celebración así como sus limitaciones. Sería un error intentar organizar un evento que citara a más participantes que aquellos que la zona fuera capaz de albergar. Igualmente, otras circunstancias tales como la previsible celebración de otro tipo de evento que limitara también la capacidad hotelera de la zona sería un handicap que habría que tener presente. Quiere esto decir que el planteamiento debería ser justamente el contrario y, en la medida de lo posible, utilizar el evento para desestacionalizar la demanda, con el cual ya se está apuntando aquí otro criterio para la selección del evento que es la no coincidencia con las épocas de temporada turística alta de la zona (Sánchez et al., 2016)

En estudios previos se observó que determinadas variables (actitud hacia el evento o satisfacción con la calidad de vida) ejercían un efecto mediador entre la percepción de los residentes sobre los impactos y el apoyo a la celebración del evento (Kaplanidou et al., 2013; Prayagt al., 2013). 
El presente trabajo pretende servir de ayuda para mejorar la gestión de los eventos deportivos, por un lado, desde el punto de vista del espectador que compra una entrada y consume un servicio deportivo en este caso un espectáculo deportivo, y por otro lado, desde el punto de vista del deportista que asiste al evento como parte integrante y participativa del engranaje organizativo del espectáculo deportivo

\section{Conclusiones}

Tras la realización del estudio sobre el impacto económico generado por la organización en Granada de la Universiada de Invierno 2015, como conclusión mas relevante es que se trata de un evento claramente rentable para la ciudad desde el punto de vista económico. Esta afirmación se encuentra refrendada por el hecho de que el impacto económico 185,66 diario medio procedente de 670 asistentes al evento lo que supone un total de 174.149 euros diarios

El nivel económico tiene su reflejo en el gasto realizado, de manera tal que la hostelería y la restauración fueron los sectores que más se beneficiaron del evento. En menor medida, también se aprovecharon los comercios y los locales de ocio
, este incremento del gasto turístico interior, al ser satisfecho, genera un aumento de la actividad (efecto directo) para los primeros proveedores de esa demanda (hoteles, restaurantes...), que deben de adecuar los pedidos a sus proveedores (alimentos, bebidas...) y éstos a su vez, para satisfacer la demanda adicional, generarán una segunda ronda de transacciones económicas, ya que deberán aumentar los pedidos a sus proveedores y así sucesivamente (efectos indirectos). Los efectos del gasto turístico interior no se agotan en los efectos directos e indirectos, pues éstos generan un incremento de las rentas de las familias vía sueldos y salarios, que se traducen en mayor consumo privado, iniciándose un nuevo ciclo de efectos que se denominan inducidos

Además de los procedimientos ya establecidos como son los retornos publicitarios, el impacto económico y la imagen de la ciudad entre otros, proponemos, además, que las organizaciones implicadas realicen estudios que determinen el grado de satisfacción de los espectadores y de los deportistas participantes en el evento.

Financiación: El presente estudio se ha financiado a través de la Fundación Empresa Universidad de Granada - EXP. No: C-3758.

\section{Referencias bibliográficas}

1. Avison Young (2003). Avison Young Olympic Impact: Vancouver 2010 and the Industrial Real Estate Market. Avison Young, Vancouver.

2. Bamford, D. \& Dehe, B. (2016) 'Service quality at the London 2012 Games - a Paralympics Athletes Survey' International Journal of Quality \& Reliability Management, 33 (2), 142-159.

3. Barajas, A., Salgado, J. y Sánchez, P. (2012). "Problemática de los estudios de impacto económico de eventos deportivos" en Estudios de Economía Aplicada, n³0: pp. 441-462.

4. Bouchet, P., Bodet, G., Bernache-Assollant, I., \& Kada, F. (2011). Segmenting sport spectators: Construction and validation of the Sport Event Experience Search (SEES) scale. Sport Management Review, 14, 42-53.

5. Brunet, Ferran (1995): “An economic analysis of the Barcelona'92 Olympic Games: resources, financing and impact", in Miquel de Moragas \& Miquel Botella, The Keys to Success: the social, sporting, economic and communications impact of Barcelona'92. Barcelona: Servei de Publicacions de la UAB, pp. 203-237.

6. Calabuig, F. y Crespo, J. (2009). Uso del método Delphi para la elaboración de una medida de calidad percibida de los espectadores de eventos deportivos. Retos: nuevas tendencias en educación física, deporte y recreación, 15, 18-24.

7. Calabuig, F., Crespo, J. y Mundina, J. (2012). Efecto del coste percibido, la calidad de servicio y la satisfacción sobre las intenciones futuras del espectador. Estudios de Economía Aplicada, 30(2), 619-636

8. Cornelissen, S. \& Swart, K.. (2006). The 2010 football world cup as a political construct: The challenge of making good on an African promise. Sociological Review, 54 (2), 108-123.

9. Crompton, J. (1995). Economic analysis of sportfacilities and events: eleven sources of misapplication. Journal of Sport Management, 9 (1) 14-35.

10. Fernández, M.T. (2014). El impacto turístico de los eventos deportivos: un estudio de caso. Cuadernos de turismo, 33, 59-76.

11. Leontef, W. (1951) Input-Output Economics. (second edition en 1986). Oxford University Press. New York.

12. Jones, C. (2001). Mega-events and host-region impacts: Determining

the true worth of the 1999 Rugby World Cup. International Journal of Tourism Research, 3: 241-151

13. Kaplanidou, K., Karadakis, K., Gibson, H., Thapa, B., Walker, M., Geldenhuys, S. (2013). Quality of Life, Event Impacts, and Mega-Event Support amongst South African Residents before and after the 2010 FIFA World Cup. Journal of Travel Research, 52(5), 631-645.

14. Mundina, J. y Calabuig, F. (2011). La Calidad de servicio percibida de un gran evento deportivo como indicador de gestión. Journal of Sports Economics \& Management, 1(1), 31-47

15. Lopes, C. y Ricci, R., (2016). Mundial FIFA 2014 en San Paulo y sus impactos: estádio arena Corinthians como equipamiento de ocio.PASOS. Revista de Turismo y Patrimonio Cultural, Octubre-Sin mes, 1241-1251.

16. OECD, 2012. Local development benefits from staging global events: achieving the local development legacy from 2012.

17. Prayag, G., Hosany, S., Nunkoo, R. y Alders, T. (2013). London residents' support for the 2012 Olympic Games: The mediating effect of overall attitude. Tourism Management, 36, 629-640

18. Pedrosa R. y Salvador, J. (2003). El impacto del deporte en la economía: problemas de medición. Revista Asturiana de Economía. 61-84.

19. Sánchez P., Fernández J. ,Salgado A.,Rodríguez A. y Barajas A. (2016). Impacto económico del XXI Campeonato de España “Open” de Invierno Master de Natación de Pontevedra 2015 Sport TK Revista euroamericana de gestión del deporte. Monográfico: Gestión y Economía del Deporte, 169-180

20. Santo, C. (2005). The economic impact of sports stadiums: Recasting the analysis in context. Journal of Urban Affairs, 27 (2), 177-191.

21. Silva, F. (2007). "Responsabilidade social do terceiro setor na mediação das parcerias públicos privados nos Megaeventos”. In: Rubio, K. (Org.). Megaeventos esportivos, legado e responsabilidade social. Casa do Psicólogo, São Paulo, 37-46.

22. Santo, C. (2005). The economic impact of sports stadiums: Recasting the analysis in context. Journal of Urban Affairs, 27 (2), 177-191. 
23. Tavares, O. (2011) "Instalacoes temporarias do Pan Rio 2007: possiveis legados” Movimento 17 (3), 11-35.

24. Theodorakis, N. \& Bebetsos, E. (2003). Examination of athletes' satisfaction: a first approach. Inquiries in Sport and Physical Education, 1(2), 197-203
25. Witt, S.F. (1988). Mega-events and mega-attractions. Tourism Management, 9 (1), 76-77.
Impacto económico de los asistentes a la Universiada de Invierno del 2015 en la ciudad... SPORT TK, 8(1), 7-12 